\title{
BMJ Open Improving institutional childbirth services in rural Southern Tanzania: a qualitative study of healthcare workers' perspective
}

\author{
Jennie Jaribu, ${ }^{1,2,3}$ Suzanne Penfold, ${ }^{4}$ Fatuma Manzi, ${ }^{3}$ Joanna Schellenberg, ${ }^{4}$ \\ Constanze Pfeiffer ${ }^{1,2}$
}

To cite: Jaribu J, Penfold S, Manzi F, et al. Improving institutional childbirth services in rural Southern Tanzania: a qualitative study of healthcare workers' perspective. BMJ Open 2016;6:e010317. doi:10.1136/bmjopen-2015010317

- Prepublication history and additional material is available. To view please visit the journal (http://dx.doi.org/ 10.1136/bmjopen-2015010317).

Received 26 October 2015 Revised 18 April 2016 Accepted 20 July 2016

\section{CrossMark}

\author{
${ }^{1}$ Swiss Tropical and Public \\ Health Institute, Basel, \\ Switzerland \\ Basel University, Basel, \\ Switzerland \\ ${ }^{3}$ Ifakara Health Institute, Dar \\ es Salaam, United Republic \\ of Tanzania \\ ${ }^{4}$ London School of Hygiene \\ and Tropical Medicine, \\ London, UK
}

Correspondence to Dr Jennie Jaribu; jennie.jaribu@unibas.ch

\section{ABSTRACT \\ Objective: To describe health workers' perceptions of a quality improvement (QI) intervention that focused on improving institutional childbirth services in primary health facilities in Southern Tanzania.}

Design: A qualitative design was applied using indepth interviews with health workers.

Setting: This study involved the Ruangwa District Reproductive and Child Health Department, 11 dispensaries and 2 health centres in rural Southern Tanzania.

Participants: 4 clinical officers, 5 nurses and 6 medical attendants from different health facilities were interviewed.

Results: The healthcare providers reported that the QI intervention improved their skills, capacity and confidence in providing counselling and use of a partograph during labour. The face-to-face QI workshops, used as a platform to refresh their knowledge on maternal and newborn health and QI methods, facilitated peer learning, networking and standardisation of care provision. The onsite follow-up visits were favoured by healthcare providers because they gave the opportunity to get immediate help, learn how to perform tasks in practice and be reminded of what they had learnt. Implementation of parallel interventions focusing on similar indicators was mentioned as a challenge that led to duplication of work in terms of data collection and reporting. District supervisors involved in the intervention showed interest in taking over the implementation; however, funding remained a major obstacle.

Conclusions: Healthcare workers highlighted the usefulness of applying a Ql approach to improve maternal and newborn health in rural settings. QI programmes need careful coordination at district level in order to reduce duplication of work.

\section{INTRODUCTION}

Antenatal care (ANC) and childbirth services in Tanzania

Tanzania faces multiple problems in delivering essential healthcare interventions such as

\section{Strengths and limitations of this study}

- This study provides in-depth understanding of healthcare workers experiences regarding the implementation of an innovative quality improvement (QI) intervention.

- It highlights benefits as well as challenges related to the implementation of the QI intervention and thus allows for important insights for scientists and also policymakers and practitioners.

- The small number of participants and the specific geographical focus limits the generalisability of the results.

- The results might have been influenced by the researcher's personal biases and idiosyncrasies.

antenatal or childbirth services. ${ }^{1-4}$ About $96 \%$ of pregnant women in Tanzania attend ANC services at least once during their pregnancy. ${ }^{5-9}$ Despite this high utilisation, the quality of preventive diagnostic and treatment services during ANC, and childbirth services are still poor. ${ }^{3}{ }^{10-12}$ Low quality of care in delivery service contributes to high maternal and neonatal mortality rates. ${ }^{13-16}$ Intrinsic factors contributing to the low quality of care include poor infrastructure, lack of skilled personnel, lack of an enabling environment for human resources and missing technical know-how for providing essential healthcare services. ${ }^{2} 1117$ Extrinsic factors include lack of funds and political commitment, lack of community participation in health-related issues and cultural beliefs. Context-specific customs, norms and beliefs play a major role in influencing healthcare-seeking behaviour, especially in rural communities. ${ }^{18} 19$

\section{Quality of care}

The Institute of Medicine defined quality of care as the degree to which health services 
for individuals and populations increase the likelihood of desired health outcomes and are consistent with current professional knowledge. ${ }^{20}$ According to the Institute of Medicine six main core needs are essential for quality of care (box 1). ${ }^{21}$

Donabedian suggested that inferences in the quality of care could be grouped into three categories: 'structure', 'process' and 'outcome'. ${ }^{22}$ 'Structure' refers to the setting where care occurs. 'process' includes what is actually done in giving and receiving care. ${ }^{23} 24$ 'Outcome' covers the effects of care on health. ${ }^{21}{ }^{25}$ In African settings a central issue for low health systems performance may be the relative neglect of healthcare processes. ${ }^{25}$ Building on these observations, a quality improvement (QI) intervention was implemented in rural Southern Tanzania aiming at improving the process of delivering antenatal and childbirth services taking into consideration three core quality needs; patient centredness, effectiveness and safety.

\section{Improving Newborn Survival in Southern Tanzania (INSIST): QI intervention}

The intervention was implemented under the umbrella of the INSIST project. ${ }^{26}$ The QI intervention used the QI approach known as the 'Collaborative Quality Improvement Model'. ${ }^{27}$ The strategy comprised of forming QI teams from different health facilities in order to create a collaborative network that is aimed at working together to achieve common goals. Twenty-three QI teams, one team per health facility, were formed each comprising a minimum of two health workers (health facility QI team) and one community representative in Ruangwa district. The community representatives were selected by the health staff and community members. They included traditional birth attendants, village leaders or community volunteers. The QI teams were responsible for testing and implementing changes and were coached and mentored by a district QI mentor. An initial pilot phase was conducted in four health facilities in Mtwara Rural district. Refer to table 1

\section{Box 1 Core needs for quality in healthcare ${ }^{21}$}

Safety. avoiding injuries to patients from the care that is intended to help them.

- Effectiveness: providing services based on scientific knowledge to all who could benefit, and refrain from providing services to those not likely to benefit.

- Patient-centred: providing care that is respectful of and responsive to individual patient preferences, needs and values, and ensuring that patient values guide all clinical decisions.

- Timely. reducing wait times and sometimes harmful delays for both those who receive and those who give care.

- Efficiency: avoiding waste, including waste of equipment, supplies, ideas and energy.

- Equitable: providing care that does not vary in quality because of personal characteristics such as gender, ethnicity, geographic location and socioeconomic status. for clear project timeline. The district QI mentor was selected by the Council Health Management Team (CHMT) and trained by the project staff on QI methods. A variety of QI methods were used to initiate the Plan-Do-Study-Act (PDSA) cycles, which formed the basis of the QI intervention. These included: identifying gaps in clinical care processes (process mapping and root cause analysis) and prioritising problems to be solved using a Pareto chart. ${ }^{28}$ This is a combined bar and line chart with the bars showing individual values in descending order and the line showing the cumulative total. The purpose of the Pareto chart is to highlight the most important points among a (typically large) set of factors. Thus it assists at identifying priorities. ${ }^{28}$ Data was used to inform the improvement process presented in the form of run charts. ${ }^{27} 29-32$

The QI teams, QI mentor and District Reproductive and Child Health (RCH) Coordinator met every 35 months in a series of five iterative workshops to ensure familiarity with the aforementioned QI tools, to participate in refresher training programmes on maternal and neonatal care services, to share their experiences and to learn from each other. Between these workshops the QI teams had activity periods where they practiced what they had learnt. Four to six weeks after each workshop, the QI teams received coaching and mentoring during on-site follow-up visits from the project staff and the QI mentor. During the visits they obtained assistance on the maternal and neonatal problems they wanted to improve, and application of QI methods. Furthermore, they were encouraged to assess their own work using data they had generated and in order to develop new ideas for improvement.

The intervention centred on two improvement objectives selected during the QI workshops by the participating healthcare providers. The first was aimed at increasing health facility deliveries through counselling on birth preparedness and pregnancy danger signs. The assumption was that improving the quality of counselling will increase the knowledge and understanding of pregnant women and allow them to make informed decisions to utilise health facilities for care, including delivery of their babies. This is important in order to avoid delay in deciding to seek care and delay in reaching the health facility. ${ }^{33}$ The second topic aimed at improving the quality of health facility deliveries by using partographs. ${ }^{34-36}$ A partograph is a graphic display of the progress of labour, which helps skilled birth attendants to recognise emerging difficulties and take action according to a clinical management protocol. ${ }^{37}$ The overall health facility deliveries improved by $46 \%$ from a median of 110 to 161 deliveries per month in a collaborative of 23 health facilities from February 2010 to June 2011. The target was to achieve a median of 220 deliveries per month. The use of partograph improved health facility deliveries by $\sim 50$ percentage points from a median of $10 \%$ to $57 \%$ in all the 23 health facilities during the same time period. 


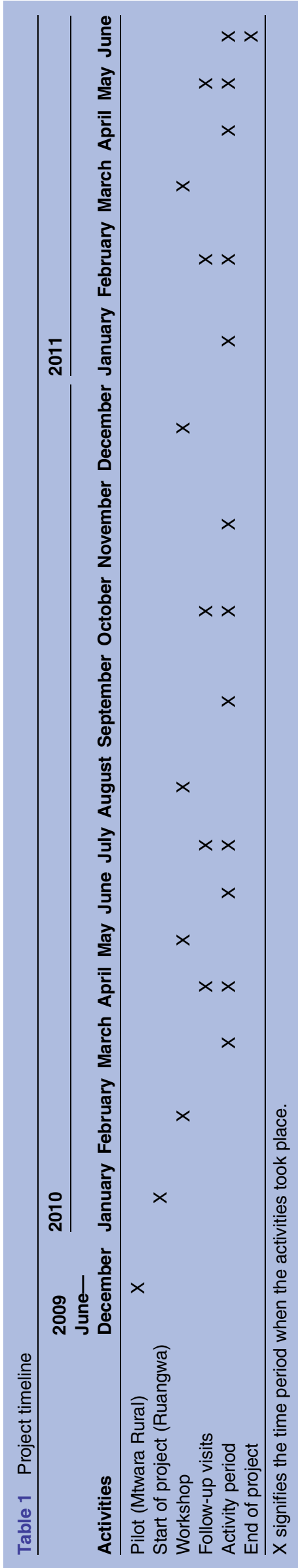

Justification of the QI improvement topics

When we started the project many of the health facilities in Ruangwa had very low to no deliveries in up to six consecutive months. As a project our original aim was to improve neonatal services. Based on the data, there were no or few neonates in the health facilities. Therefore, when we prepared Fishbone and Pareto charts, the main indication which emerged was the lack of women delivering in health facility. And this became our area of focus, in order to improve women knowledge through counselling and later improve the services they receive.

A growing number of healthcare QI interventions are implemented worldwide; increasing number of studies in low-income settings have used qualitative approaches to learn about perceptions and experiences of QI implementers. ${ }^{38-40}$ The present study is aimed at collecting evidence by conducting a qualitative study in a rural setting. We used in-depth interviews with health workers at various levels in the health system to explore their perception of the QI intervention and to identify facilitators and barriers in relation to QI implementation.

\section{METHODS}

\section{Study setting}

At the time of the study, Ruangwa district, located in Lindi Region, Southern Tanzania (figure 1), had an estimated population of 124000 with 89 registered villages. ${ }^{41}$ It was served by 24 functional health facilities, 1 district hospital, 2 health centres and 21 dispensaries. ${ }^{41}$ The QI intervention focused on the dispensaries and health centres because they are the main access point for maternal and child health services in rural areas. ${ }^{7}$ The health facilities were staffed primarily by clinical officers, nurses and medical attendants. Table 2 provides an overview of their roles and responsibilities. Each health facility offered a range of services with dispensaries having fewer services and staff compared with health centres.

\section{Data collection and analysis}

Qualitative data using in-depth interviews were collected in March 2013 by the INSIST QI project coordinator (JJ), a female medical doctor from Tanzania.

Data were collected using a multilevel approach: RCH coordinator and QI mentor at district level, and clinical officers, nurse midwives and medical attendants at health facility level. Out of the 23 health facilities that participated in the QI intervention, RCH staff from only 18 health facilities were available for interviews and 5 were not available because they were attending training outside the district. Among the available staff, health workers from five facilities were selected for pretesting the interview guides because of their accessibility and proximity to Ruangwa town. Building on the pretest, interview guides were carefully revised. One staff member from each of the 13 remaining health facilities and two representatives from the district level were 


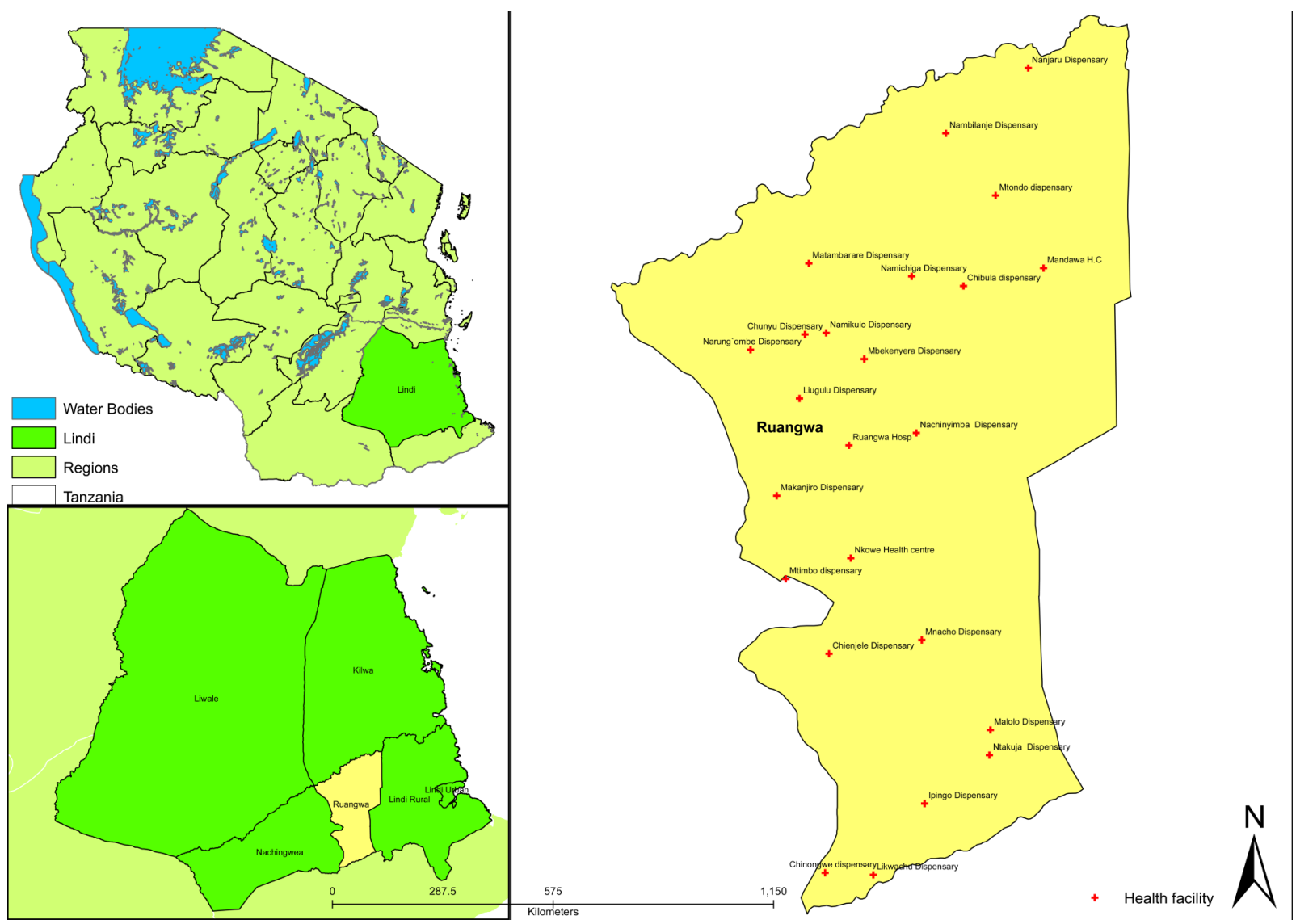

Figure 1 Map of Tanzania, Lindi and Ruangwa showing study sites at the time of the study.

Table 2 Description of healthcare provider cadres' roles and responsibilities

\begin{tabular}{|c|c|c|}
\hline $\begin{array}{l}\text { Healthcare worker } \\
\text { cadre }\end{array}$ & $\begin{array}{l}\text { Level of } \\
\text { training }\end{array}$ & Roles and responsibilities \\
\hline Clinical Officer & 3 years Diploma & $\begin{array}{l}\text { Identify and treat common diseases and perform minor surgeries } \\
\text { Participate in the planning and implementation of basic health services } \\
\text { Keep records of equipment and tools for offering services } \\
\text { Keep records, prepare and provide implementation report } \\
\text { Supervise performance of subordinate health staff }\end{array}$ \\
\hline Nurse & 3 years Diploma & $\begin{array}{l}\text { Provide nursing care to all clients in the catchment area served by their } \\
\text { facility } \\
\text { - Collect vital health statistics } \\
\text { Direct and supervise subordinate nurses } \\
\text { Provide counselling } \\
\text { Provide services to patients at home } \\
\text { - Provide preventive and childbirth services }\end{array}$ \\
\hline Medical attendant & 1-year Certificate & $\begin{array}{l}\text { Clean equipment, wards and the surrounding environment } \\
\text { Help patients with disabilities to use toilet and shower } \\
\text { To feed patients who need support } \\
\text { Take patient samples to the laboratory for testing and monitor results } \\
\text { Prepare materials for cleaning and close wounds } \\
\text { - Follow-up patients' medication requirements from drug store }\end{array}$ \\
\hline
\end{tabular}

Source: Human Resource Development Circular No. 1 of 2009 scheme of service for health worker cadres under the Tanzania Ministry of Health and Social Welfare. 
purposely selected for the interview because they were the main implementers of antenatal and childbirth services. Three types of interview guides were developed based on the role played by each member: (1) for the QI team members as implementers, (2) for the QI mentor as facilitator and (3) for the RCH coordinator as supervisor. The questions were focused on how the intervention was conducted that is, the QI process and structure and the maternal health topics (interview guides are attached in the online supplementary file). The interview guides considered topics and activities covered during the implementation of the intervention and were designed to elicit the perceptions of the study participants.

A set of 10 open-ended questions and additional relevant prompts were developed. The tools were designed in English and translated into Swahili. The translation of local terms was cross-checked with experts working in the field. The health workers were interviewed at their place of work, and the interviews took on average $45 \mathrm{~min}$ to 1 hour. Interviews were audio taped and transcribed verbatim. Data were analysed using content analysis $^{42}$ focusing on how the QI intervention was structured (PDSA cycle approach, face-to-face workshops, follow-up visits). In maternal and newborn health, we looked at the birth preparedness counselling and pregnancy danger signs, health facility deliveries and partograph use. Furthermore, we wanted to know about challenges and sustainability plans for the QI work. All transcripts were analysed with the assistance of MAXQDA software V.11, in order to identify meanings and patterns related to the QI intervention and its influence on provision of ANC. Emerging themes were coded building on discussions within the interdisciplinary project team. Three major thematic areas emerged during data analysis: (1) reflections about the QI process, (2) the contribution of the QI component regarding capacity building and (3) challenges related to the QI intervention. In addition, the first author maintained a research diary in which personal observations were noted during follow-up visits. Data from these observations complemented the findings from the in-depth interviews and allowed for triangulation and a deeper understanding of the context.

\section{Ethical considerations}

INSIST is registered on clinicaltrials.gov (NCT01022788). Written informed consent from participants was obtained before the interview and interviews were conducted in an environment where confidentially was maintained.

\section{RESULTS}

The study engaged 15 respondents, among them 11 were female, 6 were medical attendants, 5 nurses and 4 clinical officers. Their age ranged from early 20 s to late 50 s with the majority in their 30 s and $40 \mathrm{~s}$.
Ql process

PDSA cycles

On probing during the interviews 9 of the 15 respondents ( 1 clinical officer, 4 nurses and 4 medical attendants) could remember some of the key aspects of PDSA process.

We were looking for the root cause, why women do not come to deliver in health facility and then prepared a work plan for women to come to deliver in the facility.

Nurse, from a health centre

A medical attendant explaining a PDSA and QI process said,

My [QI] plan will be this, I will implement this, I will study it this way and I will decide later on how it will be. Therefore, everyone was doing this at their own health facilities. This was very helpful to plan our work and even the graphs we used to draw to assess our situation, it worked very well.

Medical attendant, from a dispensary

Two respondents (1 clinical officer and 1 nurse officer) reported using it in other aspects of their work.

I used it to improve family planning because we had a lot of underage and student pregnancies; we set a goal to reach 500 clients (new, old and defectors). We conducted health education about family planning to women. After a month we analyzed our data and we reached 600 clients.

Clinical officer, from a dispensary

All of the 15 respondents reported the QI workshops gave an opportunity to learn from, help and remind each other about technical issues. One clinical officer, two medical attendants and one nurse midwife reported that the workshops enabled them to offer standardised care, at least in terms of the messages provided during counselling and partograph use.

The community thought the changes are only implemented in our facility, but whenever different people meet and discuss, those who went to other facilities say the same thing. So they realized it's all over not only here, we did not create our own thing.

Medical attendant, from a dispensary

\section{Follow-up visits}

Fourteen respondents found the onsite follow-up visits useful to remind them of what was learnt during the workshop, provide practical assistance and to use their data to make informed decisions. The coaching and mentoring visits were regarded as being more important than the workshops because the respondents felt appreciated and noticed. Two providers commented that 
other programmes had promised to come for supervision after trainings; however, they never did so. Five health workers perceived the follow-up visits to be regular and focused. Seven respondents said the QI follow-up visits differ from CHMT supervision, mentioning that CHMT are short tempered, do not come regularly, do not solve problems and cover many things at the same time.

The follow up was not like fighting; you just explained and reminded us. Not like the one we receive from the district when someone addresses you with anger because they have a university degree.

Clinical officer, from a dispensary

Even if there will be no more workshops, it's better to visit us in our health facilities, then you realize what are our problems and help us. In addition, it motivates us, knowing that our work is seen and appreciated.

Medical attendant, from a dispensary

CHMT visits differ with what you were doing. You targeted specific topics. However, the CHMT when they come, they just look in general, "have you measured children"? "Yes". "Have you delivered babies"? "Yes". "What is going on here?" "Do you give vaccination"? "Yes". "No gas available"? "Yes". Aah OK. That's it.

Medical attendant, from a dispensary

The QI mentor explained how the INSIST QI and the CHMT worked in synergy to improve service delivery.

The follow up visits assisted the CHMT to know which health facility had shortage of equipment or supplies because we used to note facilities with problems during follow up visits. Then inform the CHMT that the following facility does not have a delivery bed or a weighing scale and the CHMT follow it up and supply the requirements. For example, they supplied a delivery bed, fridge and weighing scale to [Facility name] after we notified them.

Nurse, from the district level

\section{Partograph training}

Partograph refresher training was useful for all respondents, those who learnt it as part of their professional training (such as the clinical officers and nurse midwives) and those who never had previous training (such as medical attendants). Prior to the QI training the medical attendants did not know how to fill the partograph. Three clinical officers also acknowledged that the partograph training improved their understanding on how to fill a partograph. The nurse midwives seemed to be more competent compared with the other cadres.

I did not know how to use partograph at all, I am a Medical Attendant, when I finished school and started working, I was just told to deliver women. However, I have never used a partograph until we learnt it in this project.

Medical attendant, from a dispensary
Partograph [training] managed to remind us to monitor women in labour, and helped us to detect women who had prolonged labour or obstructed labour and made us take appropriate decision.

Nurse, from a health centre

Seven respondents reported that the intervention helped to improve referral of women in labour.

That emphasis of telling healthcare providers to refer pregnant women who have poor progress in labor has increased number of referral cases from dispensaries to district hospital. We have an ambulance, therefore when they call for it; it comes to pick up patients. And these referrals were based on induction from partograph if the labour is not progressing well.

Nurse, from the district level

However, one respondent reported challenges in using it in a resource-poor setting.

In times of power cut we cannot use partograph, we can't see. We use a torch to deliver the baby.

Nurse, from a health centre

Birth preparedness and pregnancy danger signs counselling Thirteen healthcare workers reported that they appreciated providing counselling during ANC and some emphasised differences before and after the counselling sessions in terms of increasing health facility deliveries.

Before we started a lot of women were delivering at home but I think it's because we did not provide appropriate counselling, after learning in the workshop and your follow ups in the facilities. Now we are providing counselling to women and their husbands at antenatal clinic and they come to deliver in the facility.

Medical attendant, from a dispensary

We knew about danger signs and birth plan counselling. However, when you came and emphasised its importance, then we realized aah! This is important; you know we were not used to emphasize it during health education.

Nurse, from a health centre

However, despite these counselling services cultural beliefs still play a major role in decision-making at family level especially in situations when families viewed the health problems as spiritual rather than physical in nature, influenced by their traditional understandings of disease aetiologies. A medical attendant highlighted this in her narrative below:

A young teenage couple came and reported "This is the second week we are not sleeping, my wife has difficulty in breathing as you taught us in danger signs". When I checked her, her hemoglobin level was $5 \mathrm{~g} / \mathrm{dL}$. I asked him, how much money do you have? And he said "500Tzs"; I asked him, did I not tell you about preparing 
for emergency you will need to save money? He said, "You told us but at home, they are preparing a traditional dance for her to chase away evil spirits, we sneaked out". I had to call an ambulance and referred them to the district hospital, there she received 3 units of blood transfusion and her pregnancy is still young.

Medical attendant, from a dispensary

\section{Challenges faced during the intervention}

The healthcare workers mentioned implementation of similar interventions as a major challenge to them, leading to duplication of work.

Duplication of efforts at the time INSIST implemented its QI component other interventions such as 'Mtunze Mtoto Mchanga' (INSIST community arm) and the 'Clinton Health Access Initiative' (CHAI), ${ }^{43}$ were implemented simultaneously. Seven healthcare providers reported that both these interventions aimed at increasing health facility deliveries. This brought some confusion to healthcare workers and made them question whether there was sufficient harmonisation of activities among different stakeholders. In addition, being involved in similar interventions was challenging to frontline workers in terms of time and data collection. In all these interventions they needed to collect data and provide reports on top of their routine work leading to duplication of effort. The confusion they felt about interventions was evident when respondents were asked to describe the present QI intervention during the in-depth interviews. Several respondents referred to other interventions. Four healthcare workers, for instance, highlighted the role of incentives linked to the CHAI intervention.

The community proposed a lot of things such as bicycle to go around with, registers, exercise books and uniforms for the volunteers, very nice things were given.

Clinical officer, from a dispensary

The things we did together and that with CHAI were all related because you all had one task of improving mother and newborn that is why I am confusing them. For example CHAI was providing wraps (locally known khanga), soap and nappies as incentive to women who gave birth in the health facility and INSIST community arm was teaching the same things.

Clinical officer, from a dispensary

\section{Sustainability of the intervention}

Building on the positive responses and influence of the QI intervention, the issue of continuation, however, is not decided by the healthcare workers even if they want to. The CHMT decides on interventions to be implemented at district level based on their planning and budget in the Council Comprehensive Health Plan (CCHP). However, the RCH activities have less priority compared with others such as HIV/AIDS or malaria. ${ }^{44}$ The respondent summarised these district level challenges as follows:
It was good bringing facilities together, learning from each other, but its continuation is a problem [...]. We need to put it in the budget, but when you put it, during quarterly meetings it does not pass. They say the money is not enough, very few RCH activities get approved [...]. Last year they approved only outreach services.

\section{DISCUSSION}

We found health workers' perception suggested that the QI intervention improved their capacity, their counselling skills for birth preparedness and pregnancy danger signs as well as the use of the partograph. In addition, they favoured the onsite follow-up visits over workshops, because it gave them the opportunity to learn and solve problems. Not all study participants could remember important details of the QI approach used, 21 months after their last learning session. Duplication of work was mentioned as one of the challenges due to different projects intervening in similar areas and the lack of sustainability of the intervention despite potential for improving healthcare process delivery. Similar findings were reported by a study in Rwanda rural health centres, ${ }^{45}$ that $\mathrm{QI}$ activities built confidence and capacity of health workers and that the mentorship visits were more supportive than classical district management supervision. Rowe et $a l^{46}$ conclude in their review on QI approaches that multifaceted interventions (eg, trainings and supervision) are more likely to improve performance, and that supervision with feedback was more effective than single interventions. This was also reported by the healthcare providers in our study. Most of the healthcare providers involved in the INSIST QI intervention had no previous knowledge of QI methods. However, they were motivated to engage in the intervention as they saw changes in improvement indicators taking place as a result of their efforts without using any additional resources. ${ }^{23}$ However, at the same time the risk of duplicating work for frontline health workers was identified as a challenge. ${ }^{47}$ This finding highlights the need to harmonise interventions. A study in South Africa reported harmonisation by involving various different stakeholders and creating a strong network. This network comprised of non-governmental organisations, district departments and the South African National Department of Health. By doing so they reduced duplication of work, lack of coordination and thus dilution of the intervention effects. ${ }^{48}$ In Tanzania many QI strategies are donor-funded and donor-driven projects focusing on HIV/AIDS leading to HIV-specific QI strategies that are not easily applicable in other fields especially in terms of resources and competing interest of the implementers. ${ }^{47}$ However, there is a need to integrate QI interventions in the whole health system including the maternal and newborn services. The Tanzania Ministry of Health and Social Welfare recognised the importance of building a culture of quality and institutionalising QI approaches in the healthcare system in general, through 
engaging the regions and districts. District councils should continuously invest in QI activities and integrate these in the CCHP to ensure sustainability. ${ }^{47}$ These suggestions have not yet been implemented, therefore at the moment the sustainability of QI efforts remains one of the main challenges. In line with Bardfield $e t a t^{49}$ it is argued that the sustainability of QI interventions requires development of government systems and execution of processes essential to build national QI frameworks, promote country ownership and infuse a culture of QI to build capacity at the local, regional and national levels. As much as the positive effects of the INSIST QI intervention are encouraging, the long-term effects are as yet unclear.

\section{Limitations}

The role of the project implementer as the interviewer might have influenced the way healthcare providers responded and might have felt obliged to highlight successes. However, the interviewer was aware of this and spoke with the respondents openly about her two roles prior to the interview. In addition, she dealt with it by encouraging the respondents to be open and share their positive as well as negative experiences. In addition, the contextual knowledge of the project implementer in terms of the culture, language and health systems could be advantageous.

Owing to limited resources in this study we did not ask the opinions of pregnant women or community members. Their information and that of health workers who did not participate would have provided useful additional insights. The evaluation was carried out in March 2013, and the project activities ended in June 2011, hence the results could be affected by recall bias. This was evident as some providers tended to remember activities of more recent projects. The selection of healthcare providers was restricted to those who actively participated in the QI intervention. The small number of participants and the specific geographical focus limits the generalisability of the results. However, the inputs identified add to the knowledge of implementing QI studies in rural settings and results could apply to other similar settings.

We started to conduct our study in Ruangwa district with an idea of spreading to all six INSIST study sites. However, due to limited funding we could only conduct the QI intervention in Ruangwa district. We chose to start with Ruangwa because it is the smallest among the six study districts and we wanted keep on learning about QI implementation. We focused on lower level health facilities because from our experience of working in this area, many QI intervention studies are conducted at district hospital, not giving opportunity to the lower facilities which cater to people more closely than the district hospital.

Even though we used facilities near Ruangwa town for pretesting, however, all the facilities are in rural areas and therefore, there is not much difference in terms of the context and qualifications of the healthcare providers and they all had the same QI exposure.

\section{Conclusion and recommendations}

The findings suggest that the applied QI intervention contributed to improving the quality of care of maternal and newborn health services in Southern Tanzania. Healthcare workers highlighted the potential of the problem-solving QI intervention. However, health systems capacity at district level to sustain this type of intervention is still challenging. Both sustainability and harmonisation of maternal and neonatal interventions aiming at QI need to be addressed in order to avoid duplication of work and the development of parallel systems.

Acknowledgements The authors thank all healthcare providers and district level supervisors at Ruangwa district for their participation, hard work and dedication to improve the quality of care. In addition, they would like to thank the staff of the Ifakara Health Institute-Mtwara Branch for all their support and contributions.

Contributors All the authors contributed substantially to the conception of the study, design of the manuscript, and analysis and interpretation of the data. $\mathrm{JJ}$ drafted the manuscript and SP, FM, JS and CP revised it critically for important intellectual content. All the authors provided final approval of the version to be published and agreed to be accountable for all aspects of the work in ensuring that questions related to the accuracy or integrity of any part of the work are appropriately investigated and resolved.

Funding This study was supported by the Bill and Melinda Gates Foundation through the Saving Newborn Lives Program of Save the Children, the Laerdal Foundation and UNICEF.

Disclaimer The funders had no role in the design and conduct of the study; in the collection, analysis and interpretation of the data; or in the preparation, review or approval of the manuscript.

\section{Competing interests None declared.}

Ethics approval The QI intervention formed part of the INSIST project that received ethical clearance through the National Institute of Medical Research Tanzania (NIMR/HQ/R.8c/Nol II/177), Institutional Review Board of the Ifakara Health Institute (IHRDC/ IRB/No: A 350) and the London School of Hygiene and Tropical Medicine (LSHTM; reference number A358-5316).

Provenance and peer review Not commissioned; externally peer reviewed.

Data sharing statement No additional data are available.

Open Access This is an Open Access article distributed in accordance with the Creative Commons Attribution Non Commercial (CC BY-NC 4.0) license, which permits others to distribute, remix, adapt, build upon this work noncommercially, and license their derivative works on different terms, provided the original work is properly cited and the use is non-commercial. See: http:// creativecommons.org/licenses/by-nc/4.0/

\section{REFERENCES}

1. Blank $\mathrm{A}$, Prytherch $\mathrm{H}$, Kaltschmidt J, et al. "Quality of prenatal and maternal care: bridging the know-do gap" (QUALMAT study): an electronic clinical decision support system for rural sub-Saharan Africa. BMC Med Inform Decis Mak 2013;13:44.

2. Penfold S, Shamba D, Hanson C, et al. Staff experiences of providing maternity services in rural Southern Tanzania-a focus on equipment, drug and supply issues. BMC Health Serv Res 2013;13:61.

3. Gross K, Armstrong Schellenberg J, Kessy F, et al. Antenatal care in practice: an exploratory study in antenatal care clinics in the Kilombero Valley, south-eastern Tanzania. BMC Pregnancy Childbirth 2011:11:36.

4. Pembe AB, Carlstedt A, Urassa DP, et al. Quality of antenatal care in rural Tanzania: counselling on pregnancy danger signs. $B M C$ Pregnancy Childbirth 2010;10:35.

5. National Bureau of Statistics (NBS) [Tanzania], Macro I. Tanzania demographic and health survey 2010. Dar es Salaam, Tanzania: NBS and ICF Macro, 2011. 
6. Pembe AB, Urassa DP, Carlstedt A, et al. Rural Tanzanian women's awareness of danger signs of obstetric complications. BMC Pregnancy Childbirth 2009;9:12.

7. Rockers PC, Wilson ML, Mbaruku G, et al. Source of antenatal care influences facility delivery in rural Tanzania: a population-based study. Matern Child Health J 2009;13:879-85.

8. Spangler SA, Bloom SS. Use of biomedical obstetric care in rural Tanzania: the role of social and material inequalities. Soc Sci Med 2010;71:760-8.

9. Mpembeni RN, Killewo JZ, Leshabari MT, et al. Use pattern of maternal health services and determinants of skilled care during delivery in Southern Tanzania: implications for achievement of MDG-5 targets. BMC Pregnancy Childbirth 2007;7:29.

10. Duysburgh E, Zhang WH, Ye M, et al. Quality of antenatal and childbirth care in selected rural health facilities in Burkina Faso, Ghana and Tanzania: similar finding. Trop Med Int Health 2013;18:534-47.

11. Hanson C, Ronsmans C, Penfold S, et al. Health system support for childbirth care in Southern Tanzania: results from a health facility census. BMC Res Notes 2013;6:435.

12. Spangler SA. Assessing skilled birth attendants and emergency obstetric care in rural Tanzania: the inadequacy of using global standards and indicators to measure local realities. Reprod Health Matters 2012;20:133-41.

13. Ministry of Health and Social Welfare. Women And Children First. Countdown to Ending Preventable Maternal, Newborn and Child Deaths in Tanzania, 2014.

14. van den Broek NR, Graham WJ. Quality of care for maternal and newborn health: the neglected agenda. BJOG 2009;116(Suppl 1):18-21.

15. Darmstadt GL, Kinney MV, Chopra M, et al. Who has been caring for the baby? Lancet 2014;384:174-88.

16. Austin A, Langer A, Salam RA, et al. Approaches to improve the quality of maternal and newborn health care: an overview of the evidence. Reprod Health 2014;11(Suppl 2):S1.

17. Ministry of Health and Social Welfare. The National Road Map Strategic Plan To Accelerate Reduction of Maternal, Newborn and Child Deaths in Tanzania 2008-2015: Sharpened One Plan 2014.

18. Haws RA, Mashasi I, Mrisho M, et al. "These are not good things for other people to know": how rural Tanzanian women's experiences of pregnancy loss and early neonatal death may impact survey data quality. Soc Sci Med 2010;71:1764-72.

19. Mrisho M, Schellenberg D, Manzi F, et al. Neonatal deaths in rural Southern Tanzania: care-seeking and causes of death. ISRN Pediatr 2012;2012:953401.

20. Institute of Medicine. Medicare: a strategy for quality assurance, Volume I. Washington, DC: The National Academies Press, 1990.

21. Institute of Medicine. Crossing the quality chasm: A new health system for the 21st century, 2001.

22. Donabedian A. The quality of care: How can it be assessed? JAMA 1988;260:1743-48

23. Berman J, Nkabane EL, Malope S, et al. Developing a hospital quality improvement initiative in Lesotho. Int J Health Care Qual Assur 2014;27:15-24.

24. Boucar M, Hill K, Coly A, et al. Improving postpartum care for mothers and newborns in Niger and Mali: a case study of an integrated maternal and newborn improvement programme. BJOG 2014:121(Suppl 4):127-33.

25. Heiby J. The use of modern quality improvement approaches to strengthen African health systems: a 5-year agenda. Int J Qual Health Care 2014;26:117-23.

26. Borghi J, Cousens S, Hamisi Y, et al. STUDY PROTOCOL Improving newborn survival in rural southern Tanzania: a clusterrandomised trial to evaluate the impact of a scaleable package of interventions at community level with health system strengthening, 2013.

27. Institute for Healthcare Improvement. The Breakthrough Series: IHI's Collaborative Model for Achieving Breakthrough Improvement. IHI Innovation Series white paper. Boston: Institute for Healthcare Improvement, 2003.
28. Institute for Healthcare Improvement. Pareto diagram. Secondary Pareto diagram. 2016. http://www.ihi.org/resources/Pages/Tools/ ParetoDiagram.aspx

29. USAID Health Care Improvement Project. The improvement collaborative: an approach to rapidly improve health care and scale up quality services. Bethesda, MD: USAID Health Care Improvement Project, 2008.

30. Kilo CM. A framework for collaborative improvement: lessons from the Institute for Healthcare Improvement's Breakthrough Series. Qual Manag Health Care 1998;6:1-13.

31. Benneyan JC, Lloyd RC, Plsek PE. Statistical process control as a tool for research and healthcare improvement. Qual Saf Health Care 2003;12:458-64.

32. Langley GJ, Moen R, Nolan KM, et al. The improvement guide: a practical approach to enhancing organizational performance. 2nd edn. A Wiley Imprint: Jossey Bass Wiley, 2009.

33. Thaddeus S, Maine D. Too far to walk: maternal mortality in context. Soc Sci Med 1994;38:1091-110.

34. Bosse G, Massawe S, Jahn A. The partograph in daily practice: it's quality that matters. International Journal Of Gynaecology And Obstetrics: The Official Organ Of The International Federation Of Gynaecology And Obstetrics 2002;77:243-44.

35. Gans-Lartey F, O'Brien BA, Gyekye FO, et al. The relationship between the use of the partograph and birth outcomes at Korle-Bu teaching hospital. Midwifery 2013;29:461-7.

36. Yisma E, Dessalegn B, Astatkie A, et al. Completion of the modified World Health Organization (WHO) partograph during labour in public health institutions of Addis Ababa, Ethiopia. Reprod Health 2013;10:23.

37. Ollerhead E, Osrin D. Barriers to and incentives for achieving partograph use in obstetric practice in low- and middle-income countries: a systematic review. BMC Pregnancy Childbirth 2014;14.

38. Alhassan RK, Spieker N, van Ostenberg P, et al. Association between health worker motivation and healthcare quality efforts in Ghana. Hum Resour Health 2013;11:37.

39. Afari $\mathrm{H}$, Hirschhorn LR, Michaelis A, et al. Quality improvement in emergency obstetric referrals: qualitative study of provider perspectives in Assin North District, Ghana. BMJ Open 2014;4: e005052.

40. Puchalski Ritchie LM, van Lettow M, Barnsley J, et al. Lay health workers experience of a tailored knowledge translation intervention to improve job skills and knowledge: a qualitative study in Zomba district Malawi. BMC Med Educ 2016;16:1-8.

41. Ruangwa District Council. Council Comprehensive Health Plan: July 2012-June 2013, 2012.

42. Graneheim UH, Lundman B. Qualitative content analysis in nursing research: concepts, procedures and measures to achieve trustworthiness. Nurse Educ Today 2004;24:105-12.

43. Clinton Foundation. Eliminating mother to child transmission. Secondary eliminating mother to child transmission. 2013. https:// www.clintonfoundation.org/our-work/clinton-health-access-initiative/ programs/eliminating-mother-child-transmission

44. Lawn JE, Kerber K, Enweronu-Laryea C, et al. Newborn survival in low resource settings—are we delivering? BJOG 2009;116(Suppl 1):49-59.

45. Manzi A, Magge $H$, Hedt-Gauthier BL, et al. Clinical mentorship to improve pediatric quality of care at the health centers in rural Rwanda: a qualitative study of perceptions and acceptability of health care workers. BMC Health Serv Res 2014;14:275.

46. Rowe AK, de Savigny D, Lanata CF, et al. How can we achieve and maintain high-quality performance of health workers in low-resource settings? Lancet 2005;366:1026-35.

47. Mwidunda PE, Eliakimu E. Creating a national culture of quality: the Tanzania experience. AIDS 2015;29(Suppl 2):S175-7.

48. Mate KS, Ngubane G, Barker PM. A quality improvement model for the rapid scale-up of a program to prevent mother-to-child HIV transmission in South Africa. Int J Qual Health Care 2013;25:373-80.

49. Bardfield J, Agins B, Akiyama M, et al. A quality improvement approach to capacity building in low- and middle-income countries. AIDS 2015;29(Suppl 2):S179-86. 